\title{
A LOW-NOISE BAND PASS FILTER USING AN X-BAND INJECTION- LOCKED GUNN OSCILLATOR
}

\author{
Prosenjit Bhattacharyya ${ }^{1}$, Santosh K. Dawn², Taraprasad Chattopadhyay ${ }^{3}$ \\ ${ }^{1,2,3}$ Microwave \& Optoelectronics Lab, Department of Physics, Visva-Bharati University, Santiniketan, W. B-731235, \\ India.
}

\begin{abstract}
In this paper, we report the design and implementation of a low-noise, tunable, active microwave band pass filter (BPF) which incorporates an injection-locked Gunn oscillator. The BPF has a centre frequency of $10.30 \mathrm{GHz}$ and a 3-dB bandwidth of 52 $M H z$. Calculation shows that this active BPF can reduce the input signal AM noise by at least $38 \mathrm{~dB}$. This BPF possesses signal amplification as well as signal tracking property.
\end{abstract}

Keywords: Injection-locking, Bandpass filter, X-band, Gunn oscillator, Amplitude noise.

\section{INTRODUCTION}

Microwaves span over the frequency range of $3 \mathrm{GHz}-30$ $\mathrm{GHz}$ of the electromagnetic spectrum. Sometimes it is said to cover a wider range of $0.3 \mathrm{GHz}-30 \mathrm{GHz}$. The frequency band of interest in our design is the X-band which covers the frequency range of $8 \mathrm{GHz}-12.4 \mathrm{GHz}$. Microwave bandpass filters (BPFs) are essential components of a microwave receiving system. Microwave narrowband bandpass filters (NB-BPFs) are useful in wireless communication systems be it personal wireless communication system, satellite communication using microwaves or global system mobile (GSM) communication. NB-BPFs are also used in wireless Local Area Network (LAN) making the transfer of data such as Wi-Fi, Bluetooth, 3G and 4G cellular systems and lastly in microwave radar communication.

Some of these communication systems require ultra narrow bandwidth less than $0.5 \%$. Two key parameters in narrowband (NB) BPF design are insertion loss and narrow bandwidth. There exists a trade-off between these two features. In order to achieve very narrow bandwidth, one has to accept higher insertion loss.

Various kinds of narrow band BPFs [1-8] have been reported to be designed. A narrowband microstrip BPF has been designed [5] with ground holes at L-band. Narrowband BPFs have been reported to be implemented on a coplanar waveguide (CPW) [3] configuration at S-band. Narrowband BPFs using microstrip open-loop ring resonator has been proposed and analyzed.

Microwave BPFs [1-24] can be divided into two categories, viz., passive BPF [9-13] and active BPF [14-24]. Passive $\mathrm{BPFs}$ are constructed using passive microwave components. A waveguide filter [1] is an example of this category. Frequency tuning of passive microwave filters can be achieved by varying the dimensions of resonator. The tuning time is, however, large. This is a point of inconvenience. To overcome this defect, varactor tuning of the BPFs were introduced. Later on, BPFs were constructed using MEMS technology. There is another drawback of passive microwave filter. It is its high insertion loss which originates from the low Q-value of the varactor diode.

Since the passive microwave bandpass filters (PMBPFs) suffer from the above defects, engineers designed varactor tuned active BPFs which incorporate a microwave active device. This active device is basically a differential negative resistance (DNR) device. This results in low insertion loss. The active device of the microwave active bandpass filter (MABPF) also suffers from some problems such as its stability, noise figure and intermodulation distortion. The DNR device used in the design of active BPF can be a bipolar junction transistor ( BJT), GaAs field effect transistor (FET) or high electron mobility transistor (HEMT). If the negative resistance is high then gain compensation is necessary to ensure stability of the MABPF. The noise figure of MABPF is higher than that of a PMBPF by several dB, although GaAs FETs or HEMTs can be used to have improved noise performance.

MABPFs have been designed [14-21] using microstrip coupled lines. Active BPFs in the microwave band have also been designed using MMIC technology which employ active resonator where loss is minimized by the negative resistance.

The problem of stability and noise of the MABPF has motivated the authors to design a novel MABPF which employ an X-band (8-12.4 GHz) injection-locked Gunn oscillator (ILGO) as the active device. The Gunn oscillator is a low noise device on one hand while the use of injection locking strongly reduces amplitude noise of the input signal. The overall effect is that the noise figure of our MABPF is much lower than the normal active BPFs. Secondly, the Gunn oscillator operating in the injection locked mode, is fairly stable as has been seen in our experiment. The ILGO tracks the input signal. So, our BPF is a tracking filter. 
The centre frequency of the MABPF can be tuned by varying the free-running frequency of the Gunn oscillator (GO) using a micrometer arrangement. The ILGO also acts as a power amplifier for the input signal. Thus, our design has so many advantages over the normal designs available in literature.

\section{CIRCUIT DESCRIPTION}

The experimental circuit diagram is shown in Fig. 1. A properly calibrated tunable Gunn oscillator \#1 acts as the signal source. The input $\mathrm{CW}$ microwave signal enters the magic tee and splits into two equal components through half power division at the hybrid junction which propagate down the collinear arms 2 and 3 of the magic tee. A microwave Gunn oscillator \#2 oscillates at $10.31 \mathrm{GHz}$ frequency and connected with port- 2 of the magic tee is injection locked to the input signal. The output of the locked oscillator undergoes half power division at the magic tee junction and produces a pair of identical waves which travel down the Harm (port-1) and E-arm (port-4) of the magic tee. The other wave travelling down arm-3 of the magic tee is reflected from the metallic termination at the end of this port and splits into two equi-amplitude component waves differing in phase by $\pi$ radian. One of these waves propagate down the $\mathrm{H}$-arm while the other wave travels up the E-arm of the magic tee. Thus, a pair of waves appears at the input of the Schottky diode detector connected with the E-arm of the magic tee. The composite signal is detected by the detector and the detected voltage is measured in $20 \mathrm{MHz}$ oscilloscope. The detector is not a part of the proposed BPF. It is used only to measure microwave output power by converting the input power to equivalent voltage. Some work on BPF [22, 23] and band reject filter (BRF) [24] have been done whose circuit has some similarity with the present one.

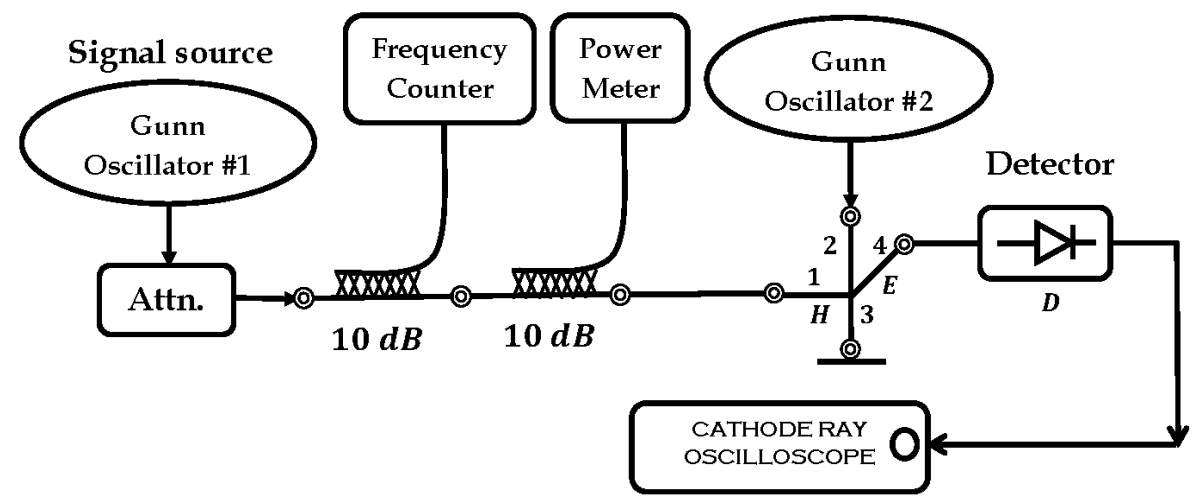

Fig. 1. Schematic circuit diagram of the experimental set up. Attn.: Variable attenuator.

\section{ANALYSIS}

We consider the input microwave signal to be frequency (or phase) modulated with a carrier frequency coinciding with the free-running frequency of the Gunn oscillator \#2 which tracks the input signal. In this case, the dc phase offset of the ILGO output relative to the input carrier will be zero. Since AM limiting takes place in the ILGO, we cannot use microwave AM signal at the input of the BPF.

In this section, we make a static analysis of the proposed low-noise active BPF. The input port of the MABPF is the port- 1 of the magic tee while port- 4 of the magic tee is the output port of the MABPF through which the output signal emerges.

Let $V_{i n}(t)=V_{0} \sin \omega_{i n} t$ be the input CW microwave signal. This signal undergoes half power division at the hybrid junction of the magic tee. The Gunn oscillator oscillates at its free running frequency $\omega_{0}$ which is connected with the collinear arm- 2 of the magic tee. The port- 3 of the magic tee is terminated by a metallic short.

The microwave signal injected into the Gunn oscillator \#2 has a voltage $V_{i n}(t) / \sqrt{2}$. The microwave injection signal power is $P_{i n j}=P_{i n} / 2$ where $P_{i n}$ is the input power entering the $\mathrm{H}$-arm of magic tee. From the theory of injection locking [9], the phase equation of the injected Gunn oscillator \#2, neglecting any asymmetry in the lock band, is given by

$$
\frac{d \psi(t)}{d t}=\frac{\omega_{0}}{2}\left(\frac{\omega_{i n}}{\omega_{0}}-\frac{\omega_{0}}{\omega_{i n}}\right)-\frac{\omega_{0}}{2 Q_{L}} \sqrt{\frac{P_{i n j}}{P_{0}}} \sin \psi(t)
$$

where $\psi(t)$ is the input-output phase error, $Q_{L}$ is the loaded quality factor and $P_{0}$ is the output power of the injection-locked Gunn oscillator \#2. In the steady state of locking $(d \psi(t) / d t)=0$. In that case,

$$
\sin \psi \approx 2 \frac{\left(\omega_{i n}-\omega_{0}\right)}{\omega_{0}} Q_{L} G
$$

where $G=\sqrt{P_{0} / P_{i n j}}$ is the locking amplitude gain. Also, $\omega_{i n} \approx \omega_{0}$ so that we have approximated $\omega_{i n}+\omega_{0} \approx 2 \omega_{0}$. The scattering matrix of the magic tee with suitable choice of the reference plane can be written as

$$
S=\frac{1}{\sqrt{2}}\left[\begin{array}{cccc}
0 & 1 & 1 & 0 \\
1 & 0 & 0 & 1 \\
1 & 0 & 0 & -1 \\
0 & 1 & -1 & 0
\end{array}\right]
$$


Since the microwave injection power, $P_{i n j}$ is comparable with the free running output power $\left(P_{f}\right)$ of the tracking GO \#2 in the present experiment, the power variation of the locked Gunn oscillator over the lockband must be taken into account. The amplitude governing equation [25] of the injected Gunn oscillator is given by

$$
\frac{d a}{d t}=a\left(1-a^{2}\right) \frac{R_{1} \omega_{0}}{2 Q_{L}}+\frac{\omega_{0}}{2 Q_{L}} \sqrt{\frac{P_{i n j}}{P_{0}}} \cos \psi
$$

where $a=\left(A / A_{0}\right), R_{1}=\left(R_{d}-R-R_{L}\right) / R_{L}$. Here, $A$ is the amplitude of the locked oscillator and $A_{0}$ is the amplitude of the free running oscillator, $-R_{d}$ is the negative resistance of the diode, $R_{L}$ is the load resistance and $R$ is the cavity resistance. In steady state of locking, $(d a / d t)=0$. In writing equation (1), the cavity resonance frequency has been assumed to be equal to the free-running frequency of the Gunn oscillator. Eliminating $\psi$ from (2) and (4) with $(d a / d t)=0$, we get the equation for amplitude variation of the locked Gunn oscillator \#2 with the detuning of the input signal frequency from the free-running Gunn oscillator frequency. This equation is expressed as

$$
\left(P_{i n j} / P_{0}\right)=a^{2}\left(1-a^{2}\right)^{2} R_{1}^{2}+\left(\frac{2 Q_{L}}{\omega_{0}}\right)^{2}\left(\omega_{i n}-\omega_{0}\right)^{2}
$$

The input microwave voltage of the diode detector connected with port-4 (i.e., the E-arm) of the magic tee is given by

$$
v_{D i n}(t)=\left(\frac{V_{0}}{2}\right) \sin \omega_{i n} t+\left(\frac{A}{\sqrt{2}}\right) \sin \left(\omega_{i n} t-\psi\right)
$$

where $A$ is the voltage amplitude of the locked Gunn oscillator \#2 output.

The input signal power response of the Schottky diode detector is shown in Fig. 2.

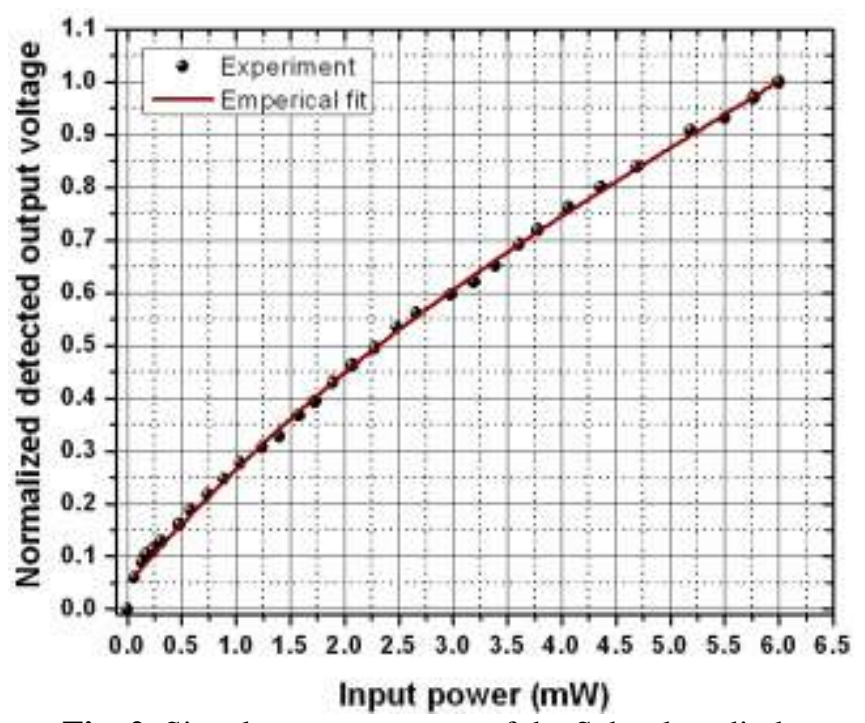

Fig. 2. Signal power response of the Schottkey diode detector. Normalizing factor for detected output voltage is $335 \mathrm{mV}$.
The output voltage of the detector is seen to follow a square root dependence with the input microwave power. Thus,

$$
\begin{aligned}
v_{\text {Dout }}^{2} \propto P_{\text {in }} & \\
& =\eta\left|v_{\text {Din }}(t)\right|^{2}
\end{aligned}
$$

where $\eta$ is the responsivity of the diode detector and $P_{i n}$ is the input microwave power to the detector. $v_{\text {Dout }}$ is maximum for $\psi=0$.

The normalized output power of the BPF is calculated as

$$
\begin{aligned}
\frac{v_{\text {Dout }}^{2}}{\left.v_{\text {Dout }}^{2}\right|_{\psi=0}}= & {\left[\frac{V_{0}^{2}+2 A^{2}+2 \sqrt{2} V_{0} A \cos \psi}{V_{0}^{2}+2 A^{2}+2 \sqrt{2} V_{0} A}\right] } \\
& =\left[\frac{1+G^{2} a^{2}+2 a G \cos \psi}{1+G^{2} a^{2}+2 G a}\right]
\end{aligned}
$$

The output power variation of the active BPF as a function of input signal frequency is described analytically by equation (8). The normalized experimental data show a close fit with the theoretical plot. The normalized factor for the experimental data is $460 \mathrm{mV}$. The $3-\mathrm{dB}$ bandwidth of the active BPF is calculated from (9) to be $50 \mathrm{MHz}$. The experimental 3-dB bandwidth is $52 \mathrm{MHz}$. The Q-value of the locked oscillator calculated from the measured lockband comes out to be 165 . Fig. 3 shows the variation of normalized output of the detector with the frequency detuning $\left(f_{\text {in }}-f_{0}\right)$.

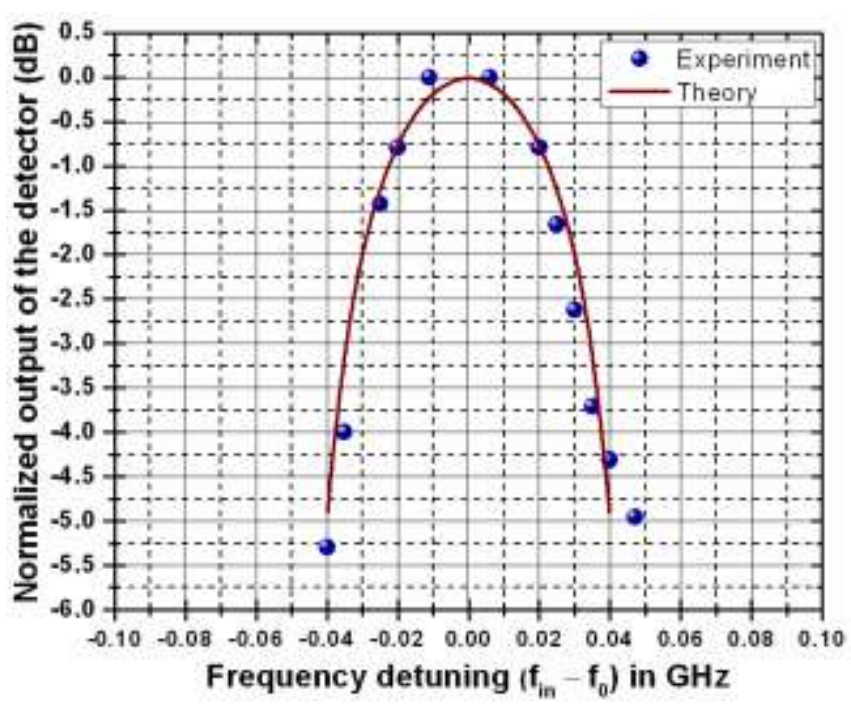

Fig. 3. Variation of normalized output of the detector with the frequency detuning $\left(f_{\text {in }}-f_{0}\right)$ in $\mathrm{GHz}$. Normalizing factor for the detector output is $460 \mathrm{mV}$.

The lockband (LB) of the Gunn oscillator \#2 is given by

$$
L B=\left(\frac{f_{0}}{Q_{L}}\right) \sqrt{\left(\frac{P_{i n j}}{P_{0}}\right)}
$$

The upper and lower lockband extremities are given by the expressions 


$$
\left(\frac{\omega_{+}}{\omega_{0}}\right)=1+\left(\frac{1}{2 Q_{L} G}\right)
$$

and

$$
\left(\frac{\omega_{-}}{\omega_{0}}\right)=1-\left(\frac{1}{2 Q_{L} G}\right)
$$

In a second experiment, we have kept the input locking signal frequency fixed at $10.34 \mathrm{GHz}$ with a power of 8.7 $\mathrm{mW}$ and varied the GO \#2 frequency over the lockband and beyond. The corresponding frequency response curve of the $\mathrm{BPF}$ is shown in Fig. 4. There is a transmission minimum between two passbands which is $8 \mathrm{~dB}$ down from the peak of the broader response. Parameter values used in numerical calculation are listed in Table-I.

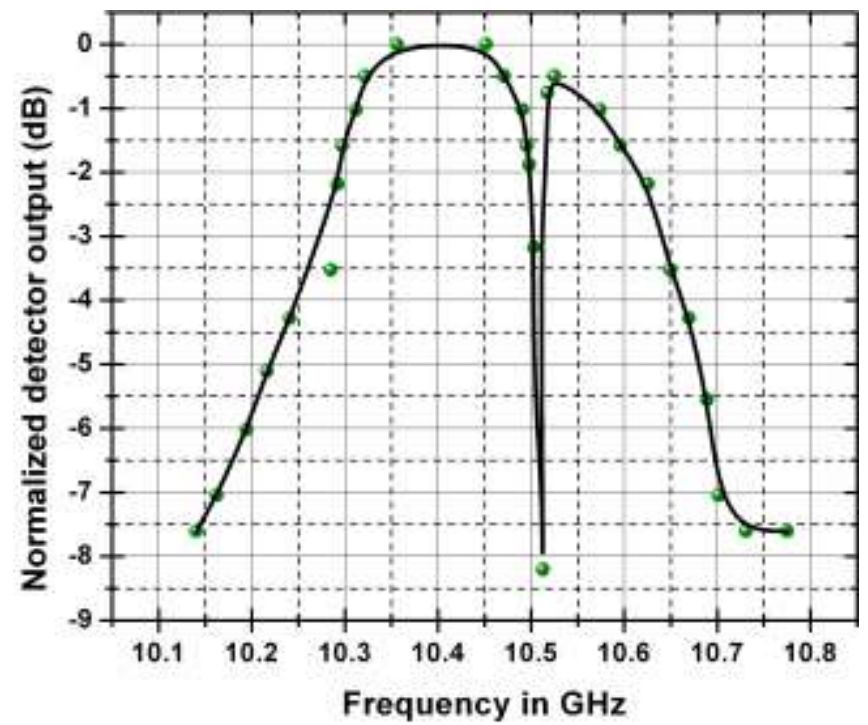

Fig. 4. Variation of detector output (in $\mathrm{dB}$ ) as a function of injected Gunn Oscillator frequency with the input locking signal frequency fixed at $10.34 \mathrm{GHz}$. Input signal power = $8.7 \mathrm{~mW}$. Normalizing factor $=3.6 \mathrm{~V}$.

\begin{tabular}{|c|c|c|}
\hline Serial No. & Parameters & Value \\
\hline 1. & Centre frequency of the BPF & $10.30 \mathrm{GHz}$ \\
\hline 2. & $\begin{array}{c}\text { Microwave injection power into slave } \\
\text { oscillator }\end{array}$ & $2.4 \mathrm{~mW}$ \\
\hline 3. & $\begin{array}{l}\text { Free-running output power of slave } \\
\text { oscillator }\end{array}$ & $3.4 \mathrm{~mW}$ \\
\hline 4. & Q-value (Experiment) & 165 \\
\hline 5. & 3-dB bandwidth of the BPF & $52 \mathrm{MHz}$ \\
\hline 6. & $\begin{array}{l}\text { Normalized resistance of Gunn } \\
\text { oscillator }\left(\mathrm{R}_{1}\right)\end{array}$ & 0.5 \\
\hline
\end{tabular}

Table I. Parameter values used in calculation

\section{REDUCTION OF AMPLITUDE NOISE BY ACTIVE BANDPASS FILTER}

In this section, we will derive a relation between the output and input power spectral densities of amplitude noise.

Let the normalized output amplitude of the injection-locked Gunn oscillator (ILGO) in presence of input amplitude noise be written as

$$
a(t)=a_{0}+a_{n}(t)
$$

where $a_{n}(t)$ is the noise term. We assume noise to be small so that $\left|a_{n}(t)\right|<<a_{0}$. Under low level injection, the output power $\left(P_{0}\right)$ of the ILGO is assumed to be nearly equal to the free-running output power $\left(P_{f}\right)$ of the Gunn Oscillator (GO).

The normalized free-running GO amplitude in presence of small noise can be expressed as

$$
a_{f}(t)=\left[1+a_{n f}(t)\right]
$$

where $a_{n f}(t)$ is the free-running GO noise term normalized by the free-running voltage amplitude in absence of noise. Here, $\left|a_{n f}\right|<<1$.

Substituting (12) and (13) in (4), we can get under low-level injection the following noise equation:

$$
\begin{aligned}
& \frac{d a_{n}(t)}{d t}+\frac{\left(1-3 a_{0}^{2}\right) R_{1} \omega_{0}}{2 Q_{L}} a_{n}(t) \\
& =\frac{\omega_{0} \cos \psi}{2 Q_{L} a_{0}}\left[v_{n i}(t)-\sqrt{\frac{P_{i n j}}{P_{f}}} a_{n f}\right]
\end{aligned}
$$

where $v_{n i}(t)$ represents input signal noise voltage normalized with respect to free-running output voltage of the locked Gunn Oscillator. Taking the Fourier transform of (14), we get a relation between the spectral density functions $F_{0}(\omega), F_{\text {in }}(\omega)$ for the output and input noise functions. Taking complex conjugate of the resulting equation and multiplying the two equations, we get a relation as

$$
S_{n o}(\omega)=\frac{\left(\frac{\omega_{0}}{2 Q_{L} a_{0}}\right)^{2} \cos ^{2} \psi}{\omega^{2}+\left(\frac{\left(1-3 a_{0}^{2}\right) R_{1} \omega_{0}}{2 Q_{L}}\right)^{2}}\left[\begin{array}{l}
S_{n i}(\omega) \\
+\frac{P_{i n j}}{P_{f}} S_{n f}(\omega)
\end{array}\right]
$$

where $\quad S_{n o}(\omega)=\left|F_{0}(\omega)\right|^{2}, \quad S_{n i}(\omega)=\left|F_{i n}(\omega)\right|^{2} \quad$ and $S_{n f}(\omega)=\left|F_{n f}(\omega)\right|^{2}$ are power spectral densities of the output and input noise, and free-running GO noise respectively. Since $Q_{L}>>1, \quad\left|\left(1-3 a_{0}^{2}\right) R_{1} / 2 Q_{L}\right|<<1$ and $a_{0} \approx 1$. Under this condition, (15) can be simplified as

$$
\frac{S_{n o}(\omega)}{S_{n i}(\omega)}=\frac{\cos ^{2} \psi}{\left(2 Q_{L} a_{0} \frac{\omega}{\omega_{0}}\right)^{2}}\left[1+\frac{P_{i n j}}{P_{f}} \frac{S_{n f}(\omega)}{S_{n i}(\omega)}\right]
$$


Eqn. (16) is solved numerically and plotted in Fig. 5 using $S_{n f} / S_{n i}$ as a parameter. The figure also implies that even in presence of GO intrinsic amplitude noise, there is a substantial reduction of amplitude noise produced by the ILGO. In numerical calculation, we have taken $\left(S_{n f} / S_{n i}\right)=$ 0,1 and 10 considering different levels of oscillator own noise.

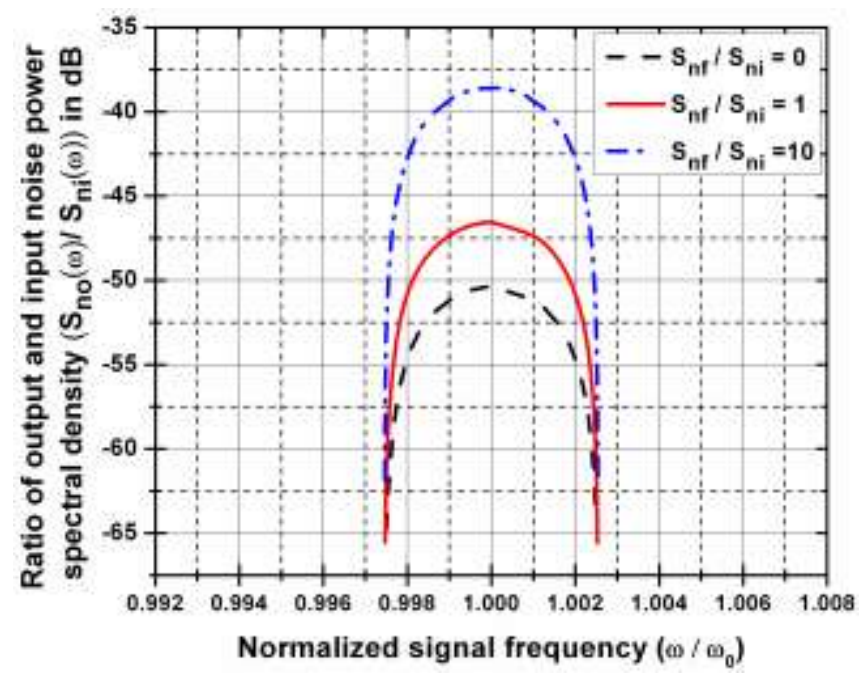

Fig. 5. Variation of ratio of output and input noise power spectral density with normalized signal frequency $\left(\omega / \omega_{0}\right)$.

\section{CONCLUSION}

A low noise, active microwave bandpass filter has been designed and operated at X-band of microwave spectrum. The BPF is tunable since the oscillation frequency of the Gunn oscillator can be varied over a specific range by varying the micrometer arrangement. This active BPF can suppress input signal AM noise better than $38 \mathrm{~dB}$ typically as predicted by the numerical calculations. This AM noise reduction is a unique property of this active BPF. This BPF can track the input signal over the lock band of the injectionlocked Gunn oscillator.

\section{REFERENCES}

[1] M. Yuceer, "Reconfigurable narrowband microwave bandpass filter," International Journal of Circuit Theory and Application, Vol. 43, pp. 125-132, 2015. Online: June, 2013.

[2] M. Hagensen, "Narrowband microwave bandpass filter design by coupling matrix synthesis," Microwave Journals, April 13, 2010.

[3] K. J. Vinoy and P. U. Reddy, "Design of narrowband bandpass filter on coplanar waveguide using spiral slots," Progress in Electromagnetic Research Letters, Vol. 6, pp. 139-148, 2009.

[4] G. Pfitzenmaier, "Synthesis and realization of narrowband canonical microwave bandpass filters exhibiting linear phase and transmission zeroes," IEEE Transaction on Microwave Theory \& Technique, Vol. 30, No. 9, Jan 2003 (Sept. 1982).
[5] J.-R. Mao, W.-W Choi, K.-W. Tam, W.-Q. Che and Q. Xue, "Tunable bandpass filter design based on external quality factor tuning and multiple mode resonators for wideband applications," IEEE Transanction on Microwave Theory Technique, vol. 61, no. 7, pp. 25742584, Jul. 2013.

[6] A. Hasan and A. E. Nadeem, "Novel microstrip hairpin line narrowband bandpass filter using via ground holes," Progress in Electromagnetic Research, PIER 78, pp. 393-419, 2008.

[7] L.-P. Zhao, X-W. Chen and C-H. Liang, "Novel design of dual-mode dual band bandpass filter with triangular resonators," Progress in Electromagnetic Research, PIER 77, pp. 417-424, 2007.

[8] B. K. Esfeh, A. Ismail, R. S. A. R. Abdullah, H. Adam and A. R. H. Alhawari, "Compact narrowband BPF using dual-mode octagonal meandered loop resonator for Wimax application," Progress in Electromagnetic Research B, Vol. 16, pp. 277-290, 2009.

[9] I. C. Hunter and J. D. Roldes, "Electronically tunable microwave band pass filters," IEEE Transaction on Microwave Theory \& Technique, Vol. 30, No. 9, pp. 1354-1360, Sept. 1982.

[10] J. Nath, D. Ghosh, J-P. Maria, A. I. Kingon, W. Fathelbale, P. D. Franzen and M. E. Stur, "An electromagnetically tunable microstrip BPF using thin film Barium Strontium Titanate (BST) varactors," IEEE Transanction on Microwave Theory \& Technique, Vol. 53, No. 9, pp. 2707-2712, Sept. 2005.

[11] M. Sanchez-Remdo, R. Gomez-Garcia, J. I. Alonse and C. B-Rodriguez, "Tunable combline filter with continuous control of centre frequency and bandwidth," IEEE Transaction on Microwave Theory \& Technique, Vol. 53, No. 1, pp. 191-199, Jan. 2005.

[12] M. Mikamoto, M. Sagawa, "Varactor tuned-band pass filters using microstrip line ring resonator," IEEE MTTs International Microwave Symposium Digest, pp. 411414, 1986.

[13]S. Kumar, "Electronically tunable ring resonator microstrip and suspended-substrate filters," Electron. Lett., vol. 27, no. 6, pp. 521-523, Mar. 1991.

[14] S. R. Chaudler, I. C. Hunter, J. G. Gardinar, "Active varactor tunable bandpass filter," Microwave \& Guided Wave Letters, vol. 3, issue. 3, pp. 70-71, Mar. 1993.

[15]B. W. Kim, Y. H. Chun and S. W. Yun, "Varactor tuned active bandpass filter with low noise performance," Electronic Letters, vol. 40, no. 15, pp. 945-946, Jul. 2004.

[16] Y. Yamamoto, Y. Imon, S. Mikumo and M. Katsurage, "Tuning a BPF by optical control of a negative resistance circuit," IEEE Transaction on Microwave Theory \& Technique, vol. 46, pp. 2006-2010, Dec. 1998.

[17] C. Y. Chang and T. Itoh, "Microwave active filters based on coupled negative resistance method," IEEE Transaction on Microwave Theory \& Technique, vol. 38, pp. 1879-1884, Dec. 1990.

[18] R. Kaunisto, K. Stadius and V. Porra, "Active MMIC filters with negative resistance compensation," Electronic Letters, Vol. 34, Issue 12, pp. 1236-1237, Jun. 1998. 
[19]D. K. Paul, M. Michael and K. Konstantinou, "MMIC tunable bandpass filter using a ring resonator with less compensation," IEEE MTT-s International Microwave Symposium Digest, pp. 941-944, 1997.

[20] K. W. Fan, C-C. Weng, Z-M. Tsai, H. Wang and S-K. Jeng, "K-band MMIC active BPFs," Microwave \& Wireless Component Letters, Vol. 15, No. 1, pp. 19-21, Jan 2005.

[21] Y. H. Chun, J-R Lee, S. W. Yun \& J-K. Rhee, "Design of a RF low noise bandpass filter using active capacitance circuit," IEEE Transaction on Microwave Theory \& Technique, Vol. 53, No. 2, pp. 687-695, Feb. 2005.

[22]S. K. Dawn and T. Chattopadhyay, "A narrowband passive microwave bandpass filter cum FM detector", Communicated.

[23] T. Chattopadhyay, P. Bhattacharyya and S. K. Dawn, "Frequency tuning of an active, microwave bandpass filter by a monotone microwave carrier," Communicated.

[24]P. Bhattacharyya, S. K. Dawn and T. Chattopadhyay, "Performance of a band reject tunable microwave filter in the face of frequency-modulated interference," Proceedings of IBSS-2015, IEEE Bombay Section Symposium-2015 (IBSS-2015), Usha Mittal Institute of Technology, Sept. 10-11, 2015.

[25] T. Chattopadhyay, "Synchronization of microwave oscillators through electrical and optical terminals," $\mathrm{Ph}$. D Thesis, Department of Physics, Burdwan University, Burdwan, India, 1988.

\section{BIOGRAPHIES}

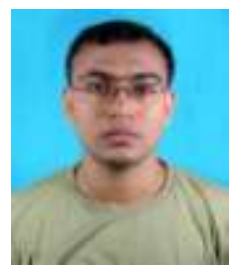

Prosenjit Bhattacharyya received his M.Sc. and $\mathrm{Ph}$. D degree from VisvaBharati University, in 2009 and 2015. He has published 24 papers in International Journals/ Conferences. His research interests include Injection-Locking technique of semiconductor laser and Microwave Filter design.

Email: prosenjit_1987@yahoo.co.in

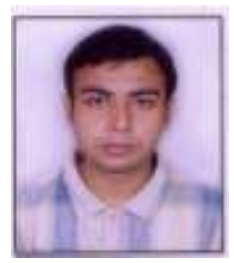

Santosh Kumar Dawn is presently pursuing his Ph.D in Microwave and Optoelectronics Lab, Department of Physics, Visva Bharati University. He received his M.Sc degree from VisvaBharati University in 2011. His topic of research is design and analysis of

Microwave Filters.

Email: santosh.dawn@gmail.com

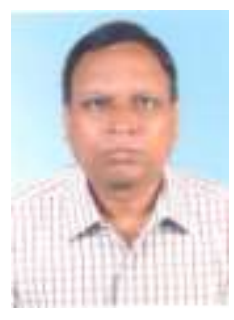

Taraprasad Chattopadhyay is a professor of Physics in Visva-Bharati (central University) since 2003. He was awarded Japanese Govt. Scholarship by the Ministry of Education and Culture, Japan.

He was an invited researcher of the Research Development Corporation, Japan and was awarded the fellowship by the association of international Education, Japan. He was a visiting professor in the Research Institute of Electrical Communication, Tohoku University, Japan. He was a professor of the Asian Institute of Technology, Bangkok, as a seconded faculty of the MHRD, Govt. of India. He was a recipient of the Networking award of the Royal society, London. He has guided and has been guiding Ph.D. students as a sole supervisor. He has conducted major and minor research projects from various funding agencies. He is a reviewer of reputed international journals of IEEE (USA), Elsevier Journals and IET(UK). He has published 114 research papers in national and international journals and conferences. He is a senior member of IEEE (USA) and a fellow of IETE (India).

\section{Email: tara_vb@hotmail.com}

